\title{
Clonal T Cell Expansion Induced by Interleukin 2 Therapy in Blood and Tumors
}

\author{
Anil Kumar, Françoise Farace, Catherine Gaudin, and Frédéric Triebel \\ Unité d'Immunologie Cellulaire, INSERM U333, Institut Gustave-Roussy, 94805 Villejuif Cedex, France
}

\begin{abstract}
In a phase I clinical trial on the effects of preoperative adjuvant IL-2 therapy given to patients undergoing hepatic resection of colorectal adenocarcinoma metastases, we monitored the putative induction of $\mathrm{T}$ cell clonal expansion in both tissues and blood. The presence of $T$ cell clonotypes was analyzed with a PCR-based method that determines V-D-J junction size patterns in T cell receptor (TCR) V $\beta$ subfamilies in samples before and after a 5-d IL-2 infusion. This high resolution method analyzing CDR3 sizes of TCR transcripts was used in conjunction with FACS ${ }^{\circledR}$ analysis of the corresponding $T$ cell subpopulations with TCR V $\beta$-specific $\mathrm{mAb}$. At time of surgery (day 8 after starting IL-2), we found in the three patients analyzed with $\mathrm{V} \beta-\mathrm{C} \beta$ primers multiple dominant $T$ cell clonotypes in the tumor and peritumoral tissues which had probably expanded as a result of therapy. In three control patients not treated with IL-2, multiple oligoclonal patterns were not observed with this set of primers. In the fourth control patient a unique $\mathrm{V} \beta 21-\mathrm{C} \beta$ CDR3 pattern which corresponds to two dominant clonotypes was found in the tumor. The same dominant clonotypes identified in the tumor after IL-2 were also detectable in the blood and comparison of the profiles obtained before and after IL-2 therapy indicates that they were induced by IL-2. The relative expansion of the corresponding $T$ cell subpopulations was maintained for varying periods of time after surgery (4-7 d and almost $2 \mathrm{yr}$ in one case). Together, these results indicate that IL-2 induces marked expansion of several T cell clones. Systemic IL-2 administration may represent, either alone or as a vaccine adjuvant, an appropriate way of boosting antigen-specific immune responses. (J. Clin. Invest. 1996. 97:1219-1226.) Key words: Interleukin $2 \cdot \mathrm{T}$ cell receptor $\bullet$ colorectal adenocarcinoma
\end{abstract}

\section{Introduction}

Immunotherapy with high doses of IL-2 demonstrates antitumor effects in some advanced cancers such as metastatic renal cell carcinoma and melanoma $(1,2)$, but toxicity is frequent and severe. As an alternative, IL-2 may be used at lower doses in an adjuvant setting, for example to improve vaccine efficacy (3), perioperatively to counteract the profound immunosuppression due to surgery and anesthesia (4), or to restore graft-

Address correspondence to F. Triebel, INSERM U333, Institut Gustave-Roussy, 39, rue Camille Desmoulins, 94805 Villejuif, France. Phone: 1-45-59-40-70; FAX: 1-45-59-49-00.

Received for publication 16 May 1995 and accepted in revised form 6 November 1995.

J. Clin. Invest.

(C) The American Society for Clinical Investigation, Inc.

0021-9738/96/03/1219/08 \$2.00

Volume 97, Number 5, March 1996, 1219-1226 versus-leukemia activity after T cell-depleted allogeneic bone marrow transplantation (5). The evaluation of future clinical trials should be facilitated by the definition of specific biological end points induced by IL-2. One of these may be the clonal expansion of tumor-specific $\mathrm{T}$ cells primed in vivo by relevant antigens (6-10).

Effective immune responses may occur physiologically in the course of tumor development. Transformed cells express tumor-associated antigens that trigger an adaptive immune response aimed at rejection of the growing cancer lesions. For instance, melanoma-associated antigenic peptides, such as those encoded by the MAGE (6), Melan-A/MART-1, MART-1 (9, 10), gp100 (8), and tyrosinase (7) genes, have been characterized and shown to be recognized by autologous cytotoxic $\mathrm{T}$ lymphocytes in vitro. Direct evidence to support this immunosurveillance concept has been reported recently in a human regressive melanoma, by showing that the regressing tumor was heavily infiltrated by tumor-specific cytotoxic $\mathrm{T}$ lymphocytes (11). In advanced cancer patients, multiple T cell clonal expansion has also been identified in either tumors or blood, including melanoma (12), renal cell adenocarcinoma (13), head and neck cancer (14), or B-chronic lymphocytic leukemia (15). It has not been determined yet whether IL-2 administration is effective in stimulating specific antitumor reaction mediated by in vivo primed $\mathrm{T}$ cells.

We initiated a phase I clinical trial on the effects of preoperative adjuvant IL-2 therapy given to patients undergoing hepatic resection of colorectal adenocarcinoma metastases (4, 16) and this provided us with an opportunity to monitor the putative induction of $\mathrm{T}$ cell clonal expansion (i.e., identification of dominant clonotypes) in tumoral or peritumoral tissues and blood. Our data show that 5-d IL-2 infusion can induce marked $\mathrm{T}$ cell clonal expansion in these patients.

\section{Methods}

Patients and samples. Three patients who underwent IL-2 therapy before surgery were studied. Patients 1 and 3 received continuous IL-2 infusion for $5 \mathrm{~d}$ at a dose of $12 \times 10^{6} \mathrm{IU} / \mathrm{m}^{2} / \mathrm{d}$ of IL-2 while patient 2 received IL-2 at a dose of $9 \times 10^{6} \mathrm{IU} / \mathrm{m}^{2} / \mathrm{d}$. Surgery was performed $48 \mathrm{~h}$ after termination of IL-2 therapy. 20-ml blood samples were collected before initiation of IL-2 therapy (day -8$)$, on the day of surgery (day 0 ), as well as 1,4 , and $7 \mathrm{~d}$ after hepatic resection. Blood was also collected from patient 2, $706 \mathrm{~d}$ after surgery. PBMC were isolated using Ficoll-Paque (Pharmacia LKB Biotechnology, Uppsala, Sweden) and frozen in 10\% DMSO in liquid nitrogen until further use.

Tumoral and nontumoral tissues were collected just after hepatic resection. Tissues were cut into small pieces (100-200 mg each), washed with $0.9 \% \mathrm{NaCl}$, and stored in liquid nitrogen for subsequent use. Hepatic tissue samples were collected from the peritumoral area and the corresponding tissue sections were found not to be invaded by tumor cells.

CDR3 size analysis. Tumoral and nontumoral tissue samples were directly squeezed (i.e., without any cell separation or in vitro culture) in $6 \mathrm{M}$ guanidium thiocyanate buffer using a Spex 6700 pulverizer (Spex Industries, Edison, NJ). Total RNA was purified by $\mathrm{CsCl}$ gradient centrifugation. For PBMC, total RNA was extracted 
using a modified guanidium thiocyanate phenol chloroform method (17). cDNA was prepared using oligo-dT priming and reverse transcriptase. The $\mathrm{V} \beta$ and $\mathrm{C} \beta$ primers have been described previously $(16,18)$. The procedure used for CDR3 size analysis, including the $\mathrm{C} \beta$-Fam and $13 \mathrm{~J} \beta$-Fam primer sequences, has also been reported $(12,15)$. Briefly, the PCR-amplified products were copied using a nested fluorescent $\mathrm{C} \beta$ or $\mathrm{J} \beta$ primer. Sizes of the fluorescent run-off products were determined by electrophoresis on an automated DNA sequencer. Since the positions of 5' and $3^{\prime}$ primers are fixed, size variations in the run-off products are only due to different CDR3 lengths, reflecting an imprecise V-D-J joining process. The peaks, at three nucleotide intervals, correspond to in-frame transcripts as described previously $(12,15)$. The graphs representing CDR3 size patterns were standardized at $100 \%$ for the highest peaks and data used to generate these graphs could be used to determine the intensity of each peak expressed as fluorescence units $(\mathrm{FU})^{1}$ and to evaluate the background. Dominant peaks were defined as high-intensity signals (e.g., from 20,000 to $100,000 \mathrm{FU}$ ) with a dramatic reduction in other CDR3 signals. The sensitivity of this assay has been tested by mixing $\mathrm{T}$ cell clones with known $\mathrm{V} \beta$ specificities with PBMC and analyzing CDR3 size patterns. The corresponding peaks were detected over the polyclonal background when a given T cell clone represented $1-5 \%$ of total PBMC.

1. Abbreviations used in this paper: FU, fluorescence units; TCR, $\mathrm{T}$ cell receptor.
Phenotypic analysis of PBMC. Immunofluorescence experiments were performed using anti-T cell receptor (TCR) $\alpha / \beta$ (BMA031), anti-CD3, -CD4, -CD8 (OKT3, OKT4, OKT8; Ortho Diagnostics, Raritan, NJ), anti-V $\beta 1,-\mathrm{V} \beta 2,-\mathrm{V} \beta 3$, -V $\beta 5$, -V $\beta 13.1,-\mathrm{V} \beta 13.6,-\mathrm{V} \beta 16$, and $-\mathrm{V} \beta 18 \mathrm{mAb}$ (Immunotech, Marseille, France).

\section{Results}

The experimental approach used here has been shown to be appropriate to readily identify a number of clonal T cell expansions in blood or tissues. It has been adapted from previous work in the mouse $(19,20)$. Two major steps are generally required to demonstrate $\mathrm{T}$ cell clonal expansion. First, the size spectrum of the CDR3-like region in each of the $24 \mathrm{~V} \beta$ subfamilies is determined using one $V \beta$ and one $C \beta$ primers. Second, given $V \beta$ subfamilies are further characterized when appropriate using $13 \mathrm{~J} \beta$-specific primers to refine the analysis. It has been shown previously either by cDNA sequencing (14, 21) or PCR analysis using clonotypic (V-D-J junction specific) primers (12) that dominant peaks obtained with only 1 out of $13 \mathrm{~V}-\mathrm{J}$ primers correspond to a single V-D-J junction (i.e., representing a clonal $\mathrm{T}$ cell subpopulation). Indeed, the use in transcripts belonging to a given $V \beta$ subfamily of a single $J \beta$ combined to a unique CDR3 size is most likely due to a single dominant transcript.

Three patients undergoing hepatic resection of colorectal adenocarcinoma metastases were given a 5-d course of preop-

\section{A}
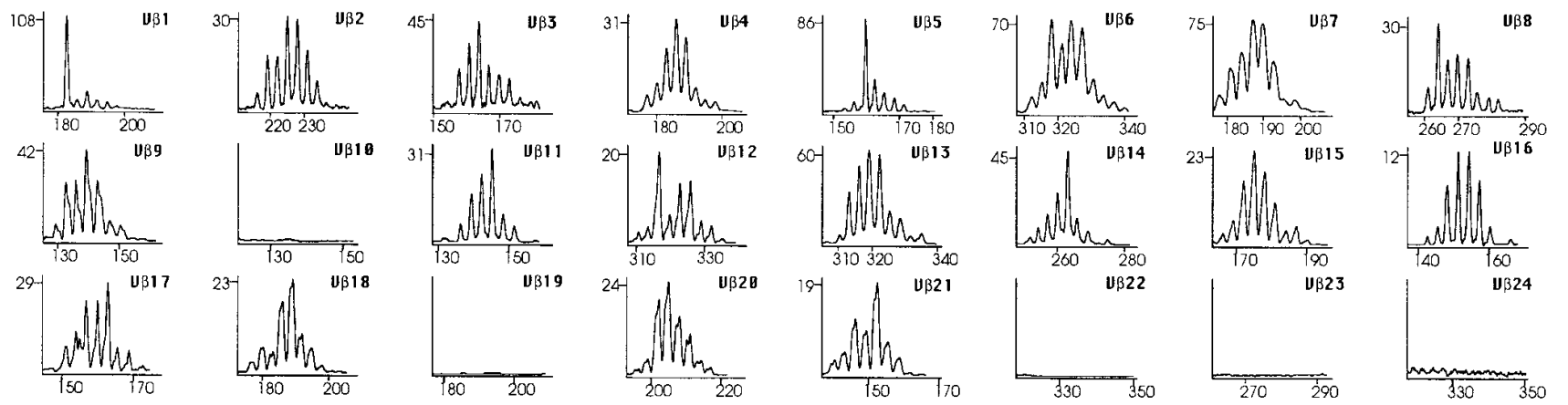

B
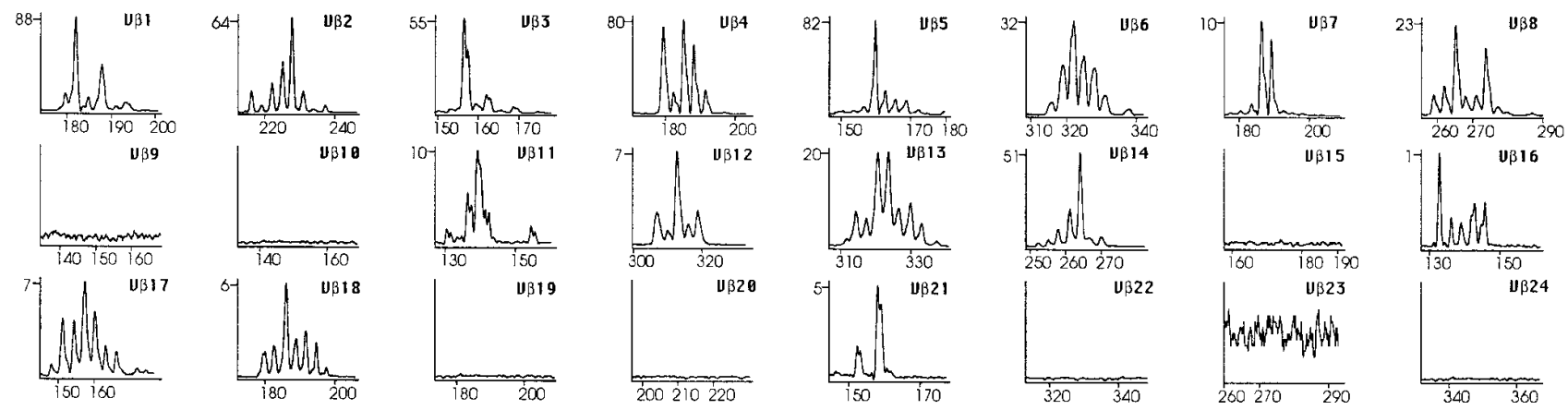

Figure 1. CDR3 size distribution in a patient receiving IL-2 therapy. RNA from PBMC $(A)$ and tumor sample $(B)$ of patient 1 at day 0 was reverse transcribed and amplified with different $\mathrm{V} \beta$ and one $\mathrm{C} \beta$ fluorescent primer. The amplified products were analyzed on an automated sequencer. The profiles obtained show size in nucleotides ( $x$-axis) and fluorescence intensity $\left(y\right.$-axis, the absolute value $\times 10^{3}$ indicates the scale) of different amplified products. 

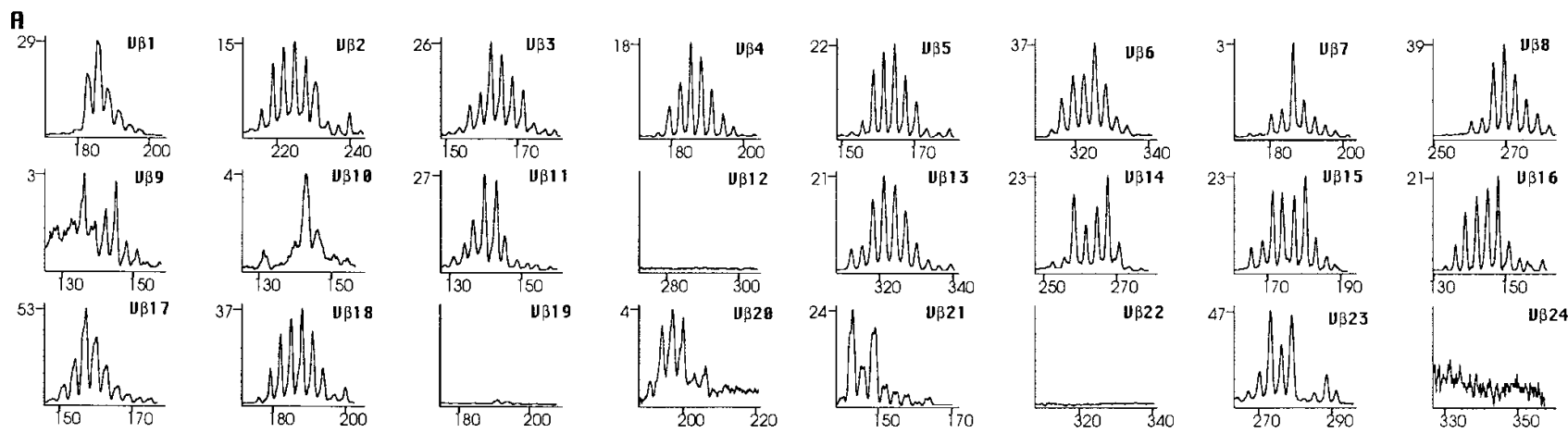
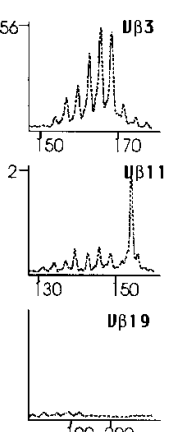

190200
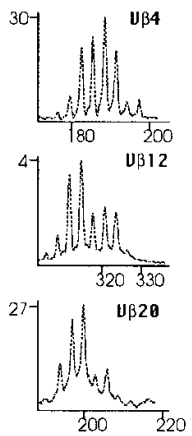
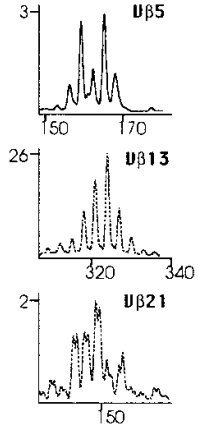
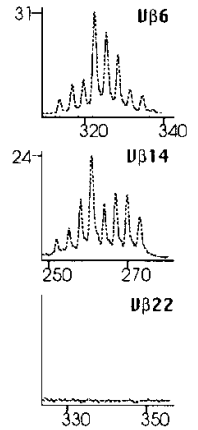
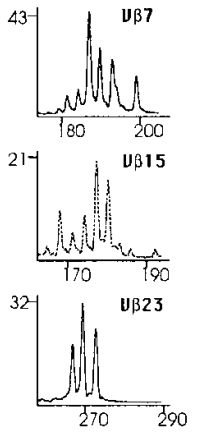

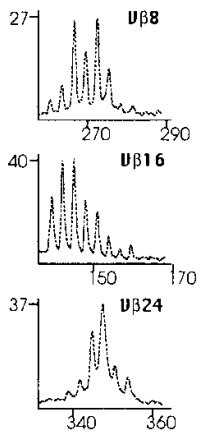

Figure 2. CDR3 size analysis in tumor of patients not treated with IL-2. Tumor RNA from patient $4(A)$ and $5(B)$ was reverse transcribed and amplified with different V $\beta$ and one $\mathrm{C} \beta$ fluorescent primer. The amplified products were analyzed on an automated sequencer. The profiles obtained show size in nucleotides ( $x$-axis) and fluorescence intensity ( $y$-axis, the absolute value $\times 10^{3}$ indicates the scale) of different amplified products.

erative adjuvant IL-2 therapy. At the time of surgery (day 0), RNA was extracted from blood, tumor, and liver, reverse transcribed, and amplified by PCR (25 cycles for PBMC, 30 cycles for tissues) using $24 \mathrm{~V} \beta$ subfamily primers and one fluorescent $\mathrm{C} \beta$-specific primer. The labeled PCR products were analyzed on an automated DNA sequencer to determine CDR3 size distribution and signal intensity. The results from patient 1's PBMCs and tumor sample are shown in Fig. 1 ( $A$ and $B$, respectively). Most blood profiles reflecting CDR3 size diversity in a given $\mathrm{V} \beta$ subfamily displayed five to eight peaks at three nucleotide intervals in a nearly Gaussian distribution. Some tumor profiles contain one or more dominant peaks, such as V $\beta 1$

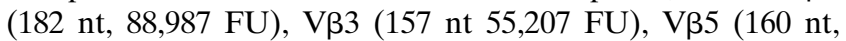
$82,760 \mathrm{FU})$, or V $\beta 14$ (264 nt, 51,985 FU) with 5-10 times more FU than the usual signal obtained in a polyclonal CDR3 size distribution. These peaks were also found in patient 1's peritumoral liver, not invaded by tumor cells (not shown). Of interest, some of the specificities such as V $\beta 1$ and V $\beta 5$ appeared as predominant peaks in PBMCs (Fig. $1 A, 108,744$ and 86,236 FU, respectively). Samples from patients 2 and 3 were analyzed in the same way, with similar results (i.e., presence of several dominant peaks, not shown). In contrast, these marked oligoclonal patterns were not observed (but once, see next paragraph) in four other patients not treated by IL-2 in this randomized (4) phase I trial. The profiles observed in the tumor and blood of two of these control patients are shown in Fig. 2 and Fig. 3, respectively. Some of the CDR3 graphs shown in Fig. 2, such as V $\beta 7$ or V $\beta 10$ in $A$ or V $\beta 11$ in $B$, are not bell-shaped. However, all graphs representing CDR3 size patterns were standardized at $100 \%$ for the highest peaks and absolute FU values have to be taken into consideration when analyzing these data. Indeed, in these cases the corresponding values were always $<5,000 \mathrm{FU}$ and therefore these signals were not interpreted as indicating dominant clonal expansion (not shown). Such altered CDR3 patterns could be observed in samples infiltrated with few $\mathrm{T}$ lymphocytes, as in the case of tumors of patients not treated with IL-2. Finally, some specificities were not detected in tumors of patients treated (Fig. 1) or not treated (Fig. 2) by IL-2 since they correspond to rare transcripts like V $\beta 19$ (which corresponds to a pseudo-gene) or the more recently described V $\beta 22$, V $\beta 23$, and V 324 subfamilies.

While marked oligoclonal patterns were not observed (but once, see below) in the four patients not treated by IL-2, clonal expansion of unique $\mathrm{T}$ cell subpopulations may nonetheless occur in the natural course of the disease. We found in the tumor of the third control patient a V $\beta 21-\mathrm{C} \beta$ profile composed of two dominant peaks (146 nt, 84,311 FU; 149 nt, 71,031 FU) and run-off products were obtained with only 2 of the $13 \mathrm{~J} \beta$ primers, J $\beta 1.5$ and $\mathrm{J} \beta 2.7$ (Fig. 4). The sizes of the latter products (105 and $109 \mathrm{nt}$ ) correspond exactly to the sizes of the $\mathrm{V}-\mathrm{C} \beta$ products, taking into account the positions of the corresponding $C \beta$ and $J \beta$ primers. Together, these data support the view that two distinct $\mathrm{V} \beta 21^{+} \mathrm{T}$ cell subpopulations were expanded at the tumor site. Of interest, the V $\beta 21-\mathrm{J} \beta 1.5$ transcript was found to be also amplified in the blood while the V $\beta 21-J \beta 2.7$ transcript was specifically amplified in tumor. The V $\beta 21-J \beta 1.5$ transcript amplification could not be suspected when analyzing the Gaussian-like $V \beta 21-C \beta$ distribution in 
A
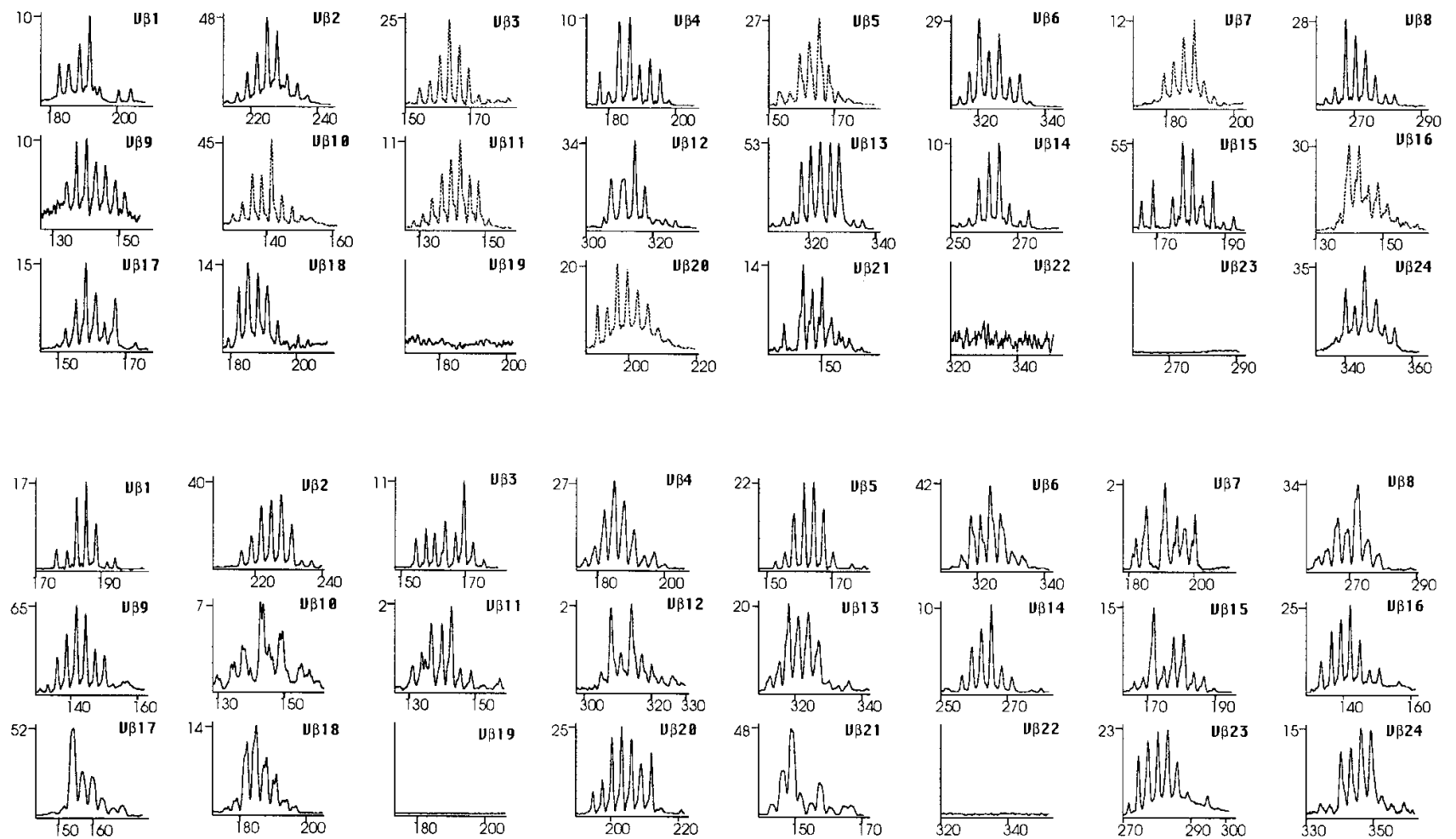

Figure 3. CDR3 size analysis in blood of patients not treated with IL-2. PBMC RNA from patient $4(A)$ and $5(B)$ was reverse transcribed and amplified with different $\mathrm{V} \beta$ and one $\mathrm{C} \beta$ fluorescent primer. The amplified products were analyzed on an automated sequencer. The profiles obtained show size in nucleotides ( $x$-axis) and fluorescence intensity ( $y$-axis, the absolute value $\times 10^{3}$ indicates the scale) of different amplified products.

PBMC and it remains to be established whether discrete $\mathrm{T}$ cell clonal expansion occurs frequently in normal individuals as well as in cancer patients.

Given the results at the time of surgery, it was interesting to evaluate whether or not the specificities displaying a dominant peak in PBMCs were induced by IL-2. We selected V $\beta 1$ and $\mathrm{V} \beta 5$ for patient $1, \mathrm{~V} \beta 16$ for patient 2 , and $\mathrm{V} \beta 20$ for patient 3 (Fig. 5). These peaks were also found in the tumor and the surrounding liver tissue (Fig. 5). These dominant clonotypes were not prevalent $8 \mathrm{~d}$ before in the blood of these patients (i.e., before IL-2 administration) with profiles showing typical CDR3 size diversity (Fig. 5), as observed in normal donors. After major surgery, anesthesia, and repetitive perioperative blood transfusions, the main clonotypes detected at the time of surgery (day 0) were still observed (Fig. 5) at day 1 in all patients and either remained predominant at day 4 and 7 (e.g., V $\beta 1$ in patient 1) or were diluted out in a more polyclonal pattern (e.g., V $\beta 5$ in patient 1).
$\boldsymbol{U} \beta 21 / \mathcal{C} \beta$
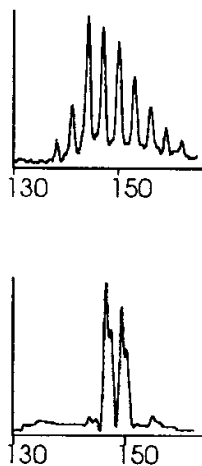

$\mathrm{U} \beta 21 / \mathrm{J} \beta 1.5$
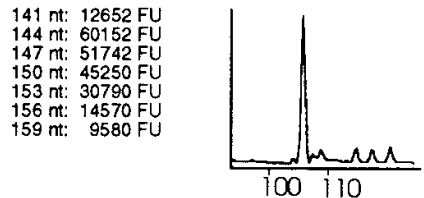

$105 \mathrm{nt}: 74908 \mathrm{FU}$

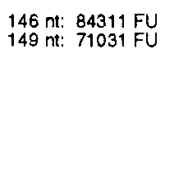

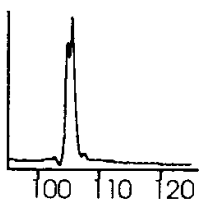

$105 \mathrm{nt}: 54216 \mathrm{FU}$

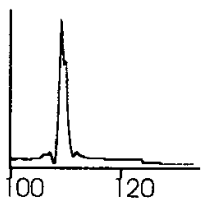

Figure 4. Clonal T cell expansion in a patient not treated with IL-2. PBMC and tumor RNA from patient 6 were reverse transcribed and amplified for 40 cycles using V $\beta 21$ and $\mathrm{C} \beta$ primers. Amplified DNA was copied for five cycles in a run-off reaction using fluorescent $C \beta$ and $\mathrm{J} \beta$ primers. The amplified products were analyzed on an automated sequencer. The profiles obtained show size in nucleotides ( $x$-axis) and fluorescence intensity ( $y$-axis) of different amplified products. 


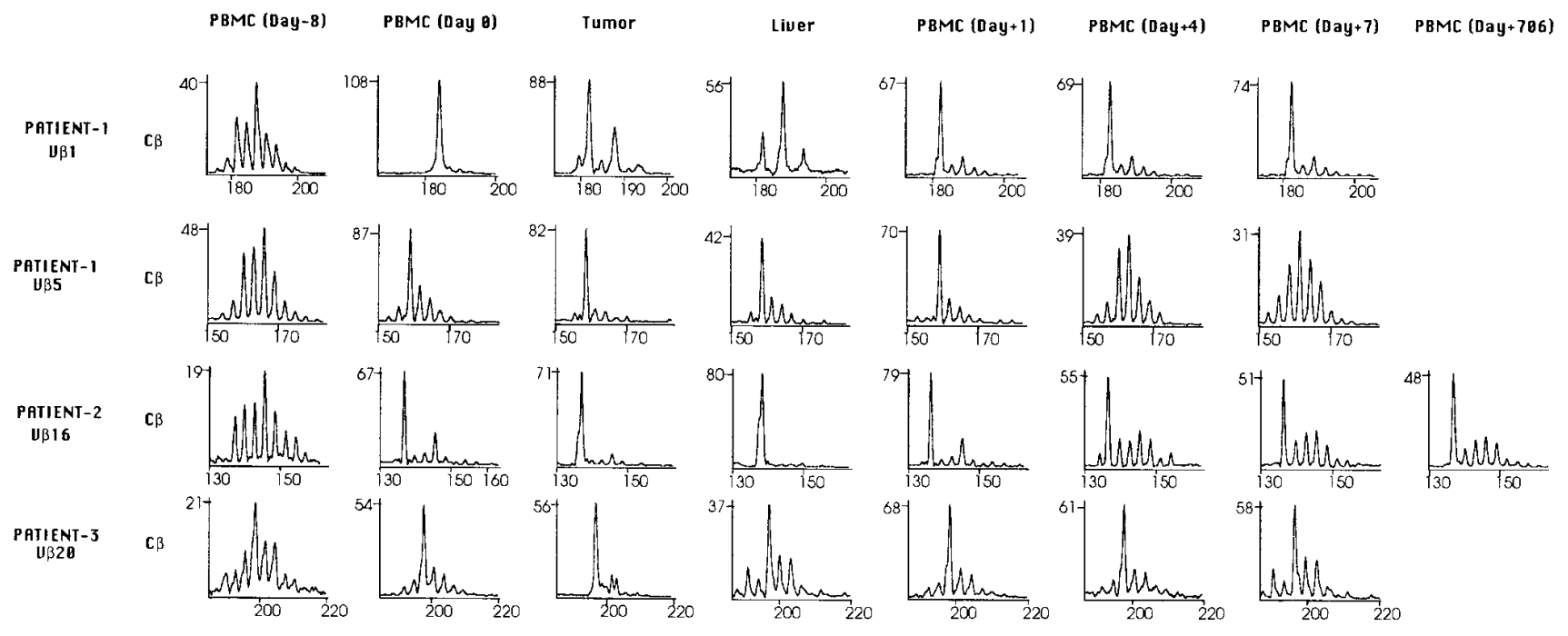

Figure 5. CDR3 size distribution of selected V $\beta$ specificities in patients treated with IL-2. RNA was extracted from PBMC collected on day -8 (before initiation of 5-d therapy with IL-2), day 0 (day of surgery), and 1, 4, and $7 \mathrm{~d}$ after surgery as well as from tumoral and liver tissue. After reverse transcription, the material was amplified with different $V \beta$ and one $C \beta$ fluorescent primer. The amplified products were analyzed on an automated sequencer. The profiles obtained show size in nucleotides ( $x$-axis) and fluorescence intensity $\left(y\right.$-axis, the absolute value $\times 10^{3}$ indicates the scale) of different amplified products.

To refine the CDR3 size analysis, the reverse-transcribed RNA was amplified using the same $V \beta$ specificity and each of the $13 \mathrm{~J} \beta$-specific primers. At the time of surgery (day 0), a bright signal was observed with only one $J \beta$ (out of 13) for three of the four $\mathrm{V} \beta$ specificities studied (V $\beta 1$ and $V \beta 5$ in patient 1 and V $\beta 20$ in patient 3) (Fig. 6). These signals appeared as single peaks, and there was exact matching between the size of these V-J $\beta$-amplified fragments (V $\beta 1-\mathrm{J} \beta 2.1,125 \mathrm{nt}$; V $\beta 5$ $\mathrm{J} \beta 1.1,117 \mathrm{nt} ; \mathrm{V} \beta 20-\mathrm{J} \beta 1.1,154 \mathrm{nt})$ and that of the predominant $\mathrm{V}-\mathrm{C} \beta$ peaks shown in Fig. 2 (V $\beta 1-\mathrm{C} \beta, 183 \mathrm{nt}$; V $\beta 5-\mathrm{C} \beta, 160 \mathrm{nt}$; $\mathrm{V} \beta 20-\mathrm{C} \beta, 198 \mathrm{nt}$ ), taking into account the positions of the corresponding $C \beta$ and $J \beta$ primers. Therefore, it is very likely that the $\mathrm{V} \beta-\mathrm{C} \beta$ peaks shown in Fig. 5 correspond to unique cells. Such marked $\mathrm{T}$ cell clonal expansion was also observed in the tumor and liver (shown in Fig. 5). Regarding the time course of such $\mathrm{T}$ cell clonal expansion, the corresponding subpopulations were still present at day 7 in blood with a progressive dilution effect on the V $\beta 5$-J $\beta 1.1$ clonotype (Fig. 6, arrow).

The $\mathrm{V} \beta 16-\mathrm{J} \beta$ profiles in patient 2 reveal a more complex situation (Fig. 6). High-intensity signals were observed for three $\mathrm{J} \beta$ specificities $(\mathrm{J} \beta 1.1, \mathrm{~J} \beta 2.1$, and $\mathrm{J} \beta 2.3)$. The size of all these three V $\beta-\mathrm{J} \beta$ DNA fragments exactly matched that of the 136-bp V $\beta 16-C \beta$-amplified fragments shown in Fig. 5, given the positions of the $\mathrm{J} \beta 1.1, \mathrm{~J} \beta 2.1$, and $\mathrm{J} \beta 2.3$ primers. One of the three $\mathrm{V} \beta-\mathrm{J} \beta$ amplified products (J $\beta 2.3)$ was also found in tumor and liver tissues (Fig. 6). This differential recruitment of $\mathrm{T}$ cell clonotypes in situ suggests that only the latter clone is tumor reactive. The two other $\mathrm{T}$ cell clones which are expanded by IL-2 in blood may have irrelevant specificities, such as viral antigen recognition.

Before IL-2 administration (day -8), an oligoclonal T cell repertoire was observed with $\mathrm{V} \beta-\mathrm{J} \beta$ PCR amplification for the four $\mathrm{V} \beta$ specificities under study $(\mathrm{V} \beta 1, \mathrm{~V} \beta 5, \mathrm{~V} \beta 16$, and $\mathrm{V} \beta 20$, see Fig. 6). In normal PBMCs, multi-peak Gaussian-like distributions are generally found with this refined CDR3 size analysis (i.e., $\mathrm{V} \beta-\mathrm{J} \beta$ amplification), even though some profiles are less smoothly shaped than those observed with $\mathrm{V} \beta-\mathrm{C} \beta$ prim- ers. In contrast to the profiles obtained with $\mathrm{V} \beta-\mathrm{C} \beta$ primers (Fig. 5), the dominant $\mathrm{V} \beta-\mathrm{J} \beta$ peaks observed at day 0 could also be identified at day -8 . Together, these results clearly support the view that IL-2 administration is capable of stimulating the growth of $\mathrm{T}$ cell clonal subpopulations, including the ones infiltrating tumors. Most $\mathrm{T}$ cell subpopulations clonally expanded by IL-2 are detectable in blood before any therapy.

Patient 2 was available for long-term follow-up. Almost 2 yr (day 706) after surgery and IL-2 administration, a V $\beta 16-C \beta$ profile, very similar to the ones at days 1,4 , and 7 after surgery, was observed with a prominent 136-nt $\mathrm{V} \beta 16-\mathrm{C} \beta$ peak (Fig. 5). Refined CDR3 size analysis with $\mathrm{J} \beta$ primers showed that only the Vß16-J $32.3 \mathrm{~T}$ cell subpopulation expanded in tumor and peritumoral tissues at day 0 was dominantly expressed at day 706 , contributing to the generation of this $136-\mathrm{nt} \mathrm{V} \beta 16-\mathrm{C} \beta$ major signal (not shown).

IL-2 induces marked changes in $\mathrm{T}$ cell subpopulation counts and we asked whether the $\mathrm{T}$ cell clonotypes identified by PCR could be detected with TCR V $\beta$-specific mAb. If so, this would indicate that the corresponding $\mathrm{T}$ cell clones (as identified by CDR3 analysis) are expanded to the point that they may be detected by cytometric analysis (limit of detection: $0.3-0.5 \%$ ). Accordingly, we quantified the corresponding $\mathrm{T}$ cell subpopulations. Results indicated that in all instances where a TCR V $\beta$-specific $\mathrm{mAb}$ was available (including all V $\beta$ specificities shown in Fig. 5) a sizable T cell subpopulation was detected after IL-2 treatment (not shown). For example, the $\mathrm{V} \beta 16^{+}$subpopulation $\left(80 \% \mathrm{CD}^{+}\right.$as assessed in dual-color immunofluorescence analysis) was identified in patient 2 before IL- 2 treatment $\left(4.2 \%\right.$ of TCR $\alpha / \beta^{+}$T cells, a polyclonal CDR3 pattern), at the time of surgery $(2.2 \%$, an oligoclonal CDR3 pattern), postsurgically at days 1 (1.3\%), 4 (2.5\%), 7 $(1.8 \%)$, and 706 after IL-2 administration (2.7\%) (Table I). Controls included other $\mathrm{V} \beta$-specific $\mathrm{mAbs}(\mathrm{V} \beta 2, \mathrm{~V} \beta 3, \mathrm{~V} \beta 5$, $\mathrm{V} \beta 13.1, \mathrm{~V} \beta 13.6)$ recognizing polyclonal $\mathrm{T}$ cell subpopulations. Variations in percentage of cells positive for a given specificity may be due to IL-2-induced changes or to the difficulty of pre- 


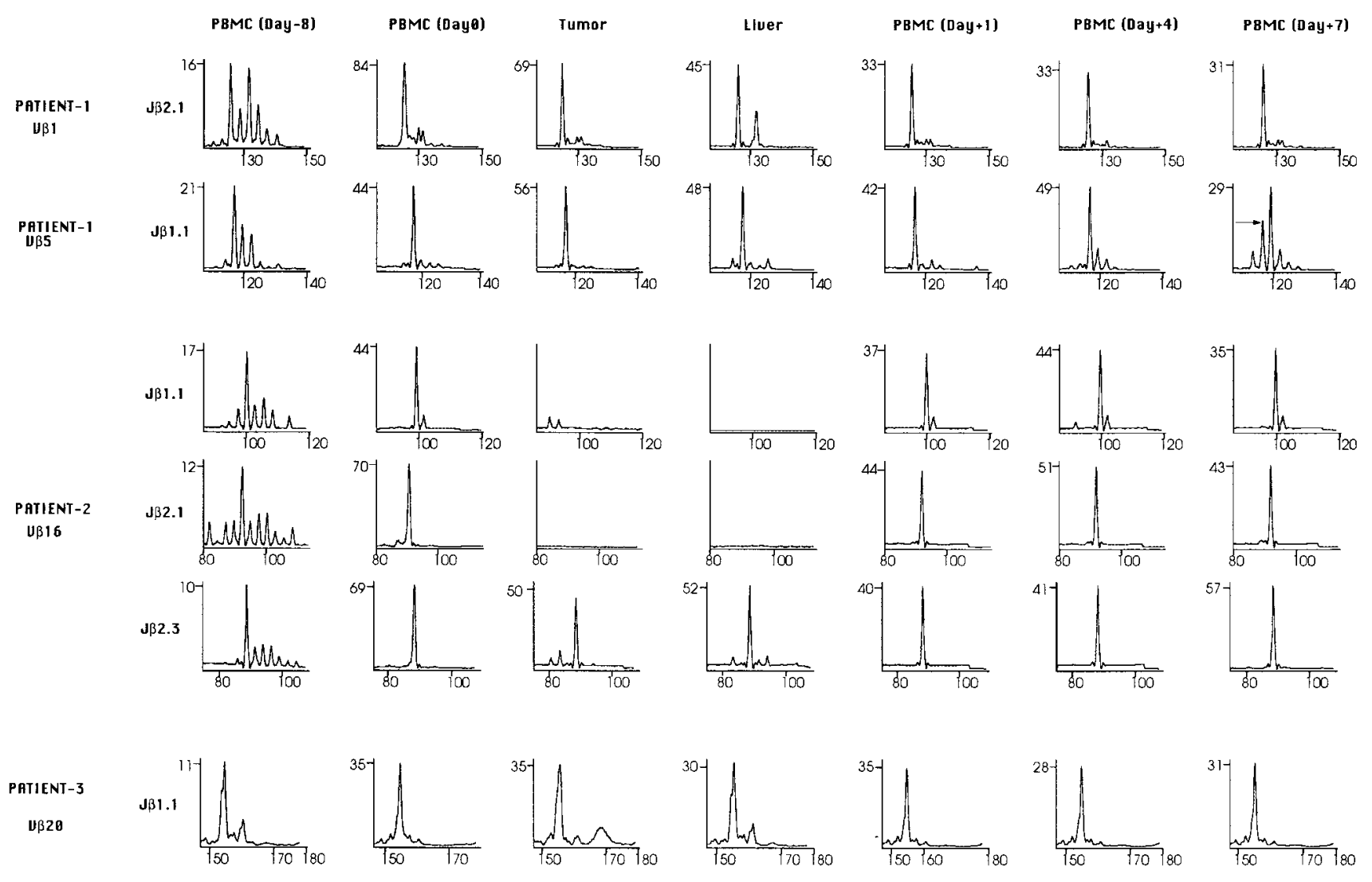

Figure 6. CDR3 size distribution of selected V $\beta$ specificities in patients treated with IL-2, as assessed with J $\beta$ primers. RNA was extracted, reverse transcribed, and amplified for 40 cycles using $\mathrm{V} \beta$ and $\mathrm{C} \beta$ primers. Amplified DNA was copied for five cycles in a run-off reaction using 13 fluorescent $\mathrm{J} \beta$ primers. The amplified products were analyzed on an automated sequencer. The profiles obtained show size in nucleotides $\left(x\right.$-axis) and fluorescence intensity ( $y$-axis, the absolute value $\times 10^{3}$ indicates the scale) of different amplified products.

cisely quantifying minor $\mathrm{T}$ cell subpopulations. Together, these results, obtained with this high-resolution CDR3 size analysis combined with FACS ${ }^{\circledR}$ analysis, indicate that marked T cell clonal expansion had occurred after IL-2 administration.

\section{Discussion}

The mechanisms underlying the therapeutic effects of IL-2 infusions are still poorly understood. The administration of highdose IL-2 results in a multitude of primary and secondary cellular events that make the immunologic evaluation of these patients extremely difficult. Indeed, many cell subsets contributing to the immune response are activated by IL-2 in vivo and these IL-2-activated cells release a series of cytokines exerting multiple biological functions. Here, we have investigated whether a single course of IL-2 infusion is able to induce T cell clonal expansion. We found multiple dominant $\mathrm{T}$ cell clonotypes in the tumors of patients treated with IL-2 in contrast to untreated patients. In addition, these clonal $\mathrm{T}$ cell subpopulations were also found in blood and their expansion was shown to be induced by IL-2.

The strong induction of clonal T cell subpopulations over a short period of time $(8 \mathrm{~d})$ combined with the fact that most of these subpopulations expanded in the tumoral and peritumoral tissues suggest that such expansion is due to the proliferation of preactivated $\mathrm{T}$ cells which may be primed by tumorassociated antigens in vivo. Indeed, in one of the four control patients not treated by IL-2 we found a transcript, V $\beta 21-\mathrm{J} \beta 2.7$, dominant in tumor but not in blood, suggesting that a specific $\mathrm{T}$ cell expansion had occurred at the tumor site, possibly after tumor-associated antigen-induced stimulation. In IL-2-treated patients, although several clonotypes were detected in the tumoral and peritumoral tissues and were therefore thought to be tumor-reactive, we have no direct evidence that these am-

Table I. Phenotypic Analysis of Patient 2's PBMC

\begin{tabular}{lcccccc}
\hline & Day -8 & Day 0 & Day +1 & Day +4 & Day +7 & Day +706 \\
\hline CD3 & 71 & 60 & 69 & 78 & 72 & ND \\
CD4 & 38 & 60 & 50 & 59 & 52 & ND \\
CD8 & 42 & 38 & 30 & 25 & 33 & ND \\
V2 & ND & 4.2 & 6.1 & ND & 4.9 & ND \\
V3 & ND & 1.4 & 0.6 & ND & 0.7 & ND \\
V5 & ND & 1.4 & 0.8 & ND & 0.9 & ND \\
V13.1 & ND & 0.8 & 0.3 & ND & 0.5 & ND \\
V13.6 & ND & 2.2 & 1.8 & ND & 2.0 & ND \\
V16 & 4.2 & 2.2 & 1.3 & 2.5 & 1.8 & 2.7 \\
& & & & & & \\
\hline
\end{tabular}

Shown are the percentages of PBMC positive for a given specificity, as determined by cytometric analysis. In the lower section, the values obtained with the TCR $\mathrm{V}$-specific $\mathrm{mAb}$ are given as percentage of $\mathrm{TCR} /^{+}$ cells (as determined using BMA031 mAb). 
plified T cell clones are tumor-specific lymphocytes. More generally, further studies are needed to determine whether such dominant $\mathrm{T}$ cell clonal expansions detected in vivo actually correspond to $\mathrm{T}$ cell clones specific for tumor cell antigens. There are to our knowledge no reports in the literature on tumor-infiltrating lymphocyte cell lines specific for colorectal adenocarcinoma. Nonetheless, it is interesting to note that such a $\mathrm{T}$ cell clone $\left(\mathrm{V} \beta 16^{+}\right)$was still detectable almost 2 yr after surgery, suggesting that it may participate in long-term tumor immune surveillance.

High-dose IL-2 administration results in lymphocytopenia followed by rebound lymphocytosis $3 \mathrm{~d}$ after IL-2 discontinuation $(15,22)$. Many CD3 lymphocytes in the blood after IL-2 therapy are $\mathrm{CD} 25^{-}$and this lymphocytosis is therefore thought to result more from profound changes in lymphocyte recirculation than from lymphocyte proliferation. IL-2-activated NK cells (i.e., expressing intermediate affinity IL-2R $\beta$ chain) are known to release a series of cytokines exerting multiple biological functions which may be responsible for the toxic effects (e.g., capillary leak syndrome) as well as the changes observed in lymphocyte recirculation. In any case, the $\mathrm{T}$ cell repertoire after IL-2 therapy was thought to be unaltered since no significant change in $\mathrm{V} \beta$ subfamily expression was found using PCR with a set of $\mathrm{V} \beta-\mathrm{C} \beta$ primers $(16,23)$. In the present study using a higher resolution PCR-based method, marked, selective T cell clonal expansion (i.e., only a few V $\beta$ specificities appeared as single peaks in CDR3 size analysis) was observed in both the blood and tissues. It was long-lasting in blood (i.e., at least $10 \mathrm{~d}$ after IL-2 discontinuation and in a V $\beta 16^{+}$clonal subpopulation lasted almost up to $2 \mathrm{yr}$ ). These $\mathrm{T}$ cell subpopulations, selected in situ and clonally amplified, are in a physical situation appropriate to contribute to tumor surveillance. They may also impair dissemination of tumor cells shed into the circulation during surgery and growth of infraclinical metastases present at the time of hepatic resection because the same T cell clones are also found amplified in the blood.

Overall, these results support the view that a single short course ( $5 \mathrm{~d})$ of IL-2 can stimulate the growth of potentially relevant (i.e., tumor-reactive) $\mathrm{T}$ cell clonal subpopulations. The possibility that long-lasting antitumor reactions which may be effective in patients with minor tumor burdens (i.e., in an adjuvant setting after surgery) may as well be induced by low-dose IL-2 therapy (e.g., given subcutaneously on an outpatient basis) remains to be investigated. Indeed, T cell clone expansion induced by IL-2 administration may well be a general finding since such an observation has been reported recently in a renal adenocarcinoma patient treated by subcutaneous low-dose IL-2 for 3 mo (24). In any case, the present study indicates that the rationale for using IL-2, either alone or as a vaccine adjuvant, in cancer patients may be to boost the preexisting antigen-specific immune reaction against tumors. This concept could also be applied to other clinical situations such as AIDS (25) or lepromatous leprosy (26).

\section{Acknowledgments}

We are grateful to Josy Vanderplancke for her expert technical assistance, to Dr. Thierry Hercend for helpful comments on the manuscript, and to Dr. Bernard Escudier and Dominique Elias for providing the blood and tumor samples.

Anil Kumar is supported by a grant from "Ligue Contre le Cancer."

\section{References}

1. Rosenberg, S.A., M.T. Lotze, J.C. Yang, P.M. Aebersold, W.M. Linehan, C. Seipp, and D.E. White. 1989. Experience with the use of high-dose interleukin-2 in the treatment of 652 cancer patients. Ann. Surg. 210:474-485.

2. Dillman, R.O., C. Church, R.K. Oldham, W.H. West, L. Schwartzberg, and R. Birch. 1993. Inpatient continuous-infusion interleukin-2 in 788 patients with cancer. Cancer (Phila.). 71:2358-2370.

3. Nohria, A., and R.H. Rubin. 1994. Cytokines as potential vaccine adjuvants. Biotherapy. 7:261-269.

4. Elias, D., F. Farace, F. Triebel, J.M. Hatchouel, J.P. Pignon, A. Lecesne, P. Rougier, P. Lasser, P. Duvillard, and B. Escudier. 1995. Phase I-II randomized study on prehepatectomy rIL-2 immunotherapy in patients with metastatic colorectal cancer. J. Am. Coll. Surg. 181:303-310.

5. Soiffer, R.J., C. Murray, R. Gonin, and J. Ritz. 1994. Effect of low-dose interleukin-2 on disease relapse after T-cell-depleted allogeneic bone marrow transplantation. Blood. 84:964-971.

6. Van der Bruggen, P., C. Traversari, P. Chomez, C. Lurquin, E. De Plaen, B. Van den Eynde, A. Knuth, and T. Boon. 1991. A gene encoding an antigen recognized by cytolytic T lymphocytes on a human melanoma. Science (Wash. DC). 254:1643-1647.

7. Brichard, V., A. Van Pel, T. Wolfel, C. Wolfel, E. Deplaen, B. Lethe, P. Coulie, and T. Boon. 1993. The tyrosinase gene codes for an antigen recognized by autologous cytolytic T lymphocytes on HLA-A2 melanomas. J. Exp. Med. 178:489-495.

8. Kawakami, Y., S. Eliyahu, C.H. Delgado, P.F. Robbins, K. Sakaguchi, E. Appella, J.R. Yannelli, G.J. Adema, T. Miki, and S.A. Rosenberg. 1994. Identification of a human melanoma antigen recognized by tumor-infiltrating lymphocytes associated with in vivo tumor rejection. Proc. Natl. Acad. Sci. USA. 91:6458-6462.

9. Kawakami, Y., S. Eliyahu, C.H. Delgado, P.F. Robbins, L. Rivoltini, S.L. Topalian, T. Miki, and S.A. Rosenberg. 1994. Cloning of the gene coding for a shared human melanoma antigen recognized by autologous $\mathrm{T}$ cells infiltrating into tumor. Proc. Natl. Acad. Sci. USA. 91:3515-3519.

10. Coulie, P.G., V. Brichard, A. Van Pel, T. Wolfel, J. Schneider, C. Traversari, S. Mattei, E.D. Plaen, C. Lurquin, J.P. Szikora, et al. 1994. A new gene coding for a differentiation antigen recognized by autologous cytolytic $\mathrm{T}$ lymphocytes on HLA-A2 melanomas. J. Exp. Med. 180:35-42.

11. Mackensen, A., G. Carcelain, S. Viel, M.C. Raynal, H. Michalaki, F. Triebel, J. Bosq, and T. Hercend. 1994. Direct evidence to support the immunosurveillance concept in a human regressive melanoma. J. Clin. Invest. 93:13971402.

12. Puisieux, I., J. Even, C. Pannetier, F. Jotereau, M. Favrot, and P. Kourilsky. 1994. Oligoclonality of tumor-infiltrating lymphocytes from human melanomas. J. Immunol. 153:2807-2818.

13. Gaudin, C., P.Y. Dietrich, S. Robache, M. Guillard, B. Escudier, M.J. Terrier-Lacombe, A. Kumar, F. Triebel, and A. Caignard. 1995. In vivo local expansion of clonal T cell subpopulations in renal cell carcinoma. Cancer Res. 55:685-690.

14. Caignard, A., P.Y. Dietrich, V. Morand, A. Lim, C. Pannetier, A.M. Leirdant, T. Hercend, J. Even, P. Kourilsky, and F. Triebel. 1994. Evidence for T-cell clonal expansion in a patient with squamous cell carcinoma of the head and neck. Cancer Res. 54:1292-1297.

15. Farace, F., F. Orlanducci, P.Y. Dietrich, C. Gaudin, E. Angevin, M.H. Courtier, C. Bayle, T. Hercend, and F. Triebel. 1994. T cell repertoire in patients with B-chronic lymphocytic leukemia: evidence for multiple in vivo $\mathrm{T}$ cell clonal expansions. J. Immunol. 153:4281-4290.

16. Savelieva, E., F. Farace, E. Angevin, D. Elias, B. Escudier, P. Duvillard, T. Hercend, and F. Triebel. 1994. T-cell receptor repertoire in colorectal adenocarcinoma patients with hepatic metastases and its changes induced by preoperative adjuvant interleukin-2 therapy. J. Immunother. 16:66-76.

17. Belyavsky, A., T. Vinogradova, and K. Rajewsky. 1989. PCR-based cDNA library construction: general cDNA libraries at the level of a few cells. Nucleic Acids Res. 17:2919-2932.

18. Genevee, C., A. Diu, J. Nierat, A. Caignard, P.Y. Dietrich, L. Ferradini, S. Roman-Roman, F. Triebel, and T. Hercend. 1992. An experimentally validated panel of subfamily-specific oligonucleotide primers (V $\alpha 1-w 29 / V \beta 1-w 24)$ for the study of human $\mathrm{T}$ cell receptor variable $\mathrm{V}$ gene segment usage by polymerase chain reaction. Eur. J. Immunol. 22:1261-1269.

19. Cochet, M., C. Pannetier, A. Regnault, S. Darche, C. Leclerc, and P. Kourilsky. 1992. Molecular detection and in vivo analysis of the specific T cell response to a protein antigen. Eur. J. Immunol. 22:2639-2647.

20. Pannetier, C., M. Cochet, S. Darche, A. Casrouge, M. Zoller, and P. Kourilsky. 1993. The sizes of the CDR3 hypervariable regions of the murine T-cell receptor $\beta$ chains vary as a function of the recombined germ-line segments. Proc. Natl. Acad. Sci. USA. 90:4319-4323.

21. Dietrich, P.Y., A. Caignard, A. Lim, V. Chung, J.L. Pico, C. Pannetier, P. Kourilsky, T. Hercend, J. Even, and F. Triebel. 1994. In vivo T-cell clonal amplification at time of acute graft-versus-host disease. Blood. 84:2815-2820.

22. Lotze, M.T., E.A. Grimm, A. Mazumder, J.L. Strausser, and S.A Rosenberg. 1981. In vitro growth of cytotoxic human lymphocytes. Lysis of fresh and cultured autologous tumor by human lymphocytes cultured in T-cell 
growth factor. Cancer Res. 41:4420-4425.

23. Farace, F., E. Angevin, B. Escudier, A. Caignard, P.Y. Dietrich, C. Genevee, T. Hercend, and F. Triebel. 1993. Influence of interleukin-2 administration on the expression of T-cell receptor $\mathrm{V}$ gene segments in patients with renal-cell carcinoma. Int. J. Cancer. 54:741-747.

24. Farace, F., E. Angevin, P.-Y. Dietrich, C. Leboullaire, J. Vanderplancke, B. Escudier, and F. Triebel. 1995. Low-dose IL-2 treatment: activation of discrete T- and NK-cell sub-populations in vivo. Int. J. Cancer. 62:523-528.

25. Teppler, H., G. Kaplan, K.A. Smith, A.L. Montana, P. Meyn, and Z.A.
Cohn. 1993. Prolonged immunostimulatory effect of low-dose polyethylene glycol interleukin 2 in patients with human immunodeficiency virus type 1 infection. J. Exp. Med. 177:483-492.

26. Kaplan, G., R. Kiessling, S. Teklemariam, G. Hancock, G. Sheftel, C.K Job, P. Converse, T.H.M. Ottenhoff, M. Becx-Bleumink, M. Dietz, and Z.A Cohn. 1989. The reconstitution of cell-mediated immunity in the cutaneous lesions of lepromatous leprosy by recombinant interleukin-2. J. Exp. Med. 169: 893-907. 